\title{
Patrones de cierre velofaringeo: Estudio comparativo entre población sana y pacientes con paladar hendido
}

\section{Patterns of velopharyngeal closure: comparative study between healthy population and patients with cleft palate}

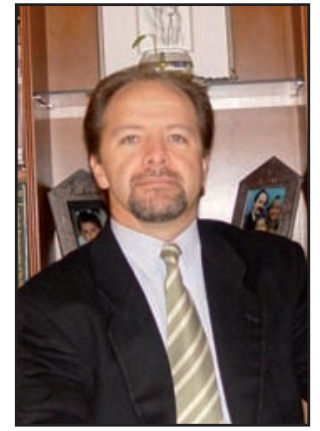

Prada Madrid, J.R.

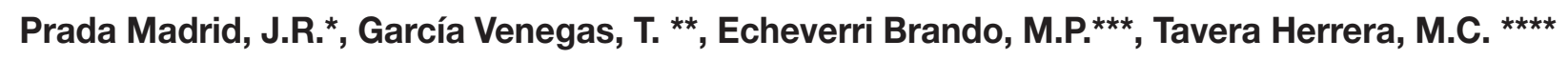

\section{Resumen}

El propósito de este estudio es corroborar los patrones de cierre velofaríngeo descritos en la literatura y su frecuencia de aparición en una muestra no probabilística de voluntarios sanos, así como comparar estos hallazgos con los obtenidos en pacientes con paladar fisurado reparado quirúrgicamente.

Diseñamos para ello un estudio descriptivo de corte transversal. Las valoraciones se realizaron en un hospital de cuarto nivel y en el Centro de Rehabilitación para Niños con Labio y Paladar Fisurado (FISULAB), en santa Fe de Bogotá (Colombia)

Participaron en el estudio 100 voluntarios sanos estudiantes universitarios y 82 pacientes con paladar hendido reparado quirúrgicamente.

Realizamos videonasofaringoscopia para determinar los patrones de cierre velofaríngeo presentes y su frecuencia de aparición.

El análisis estadístico se realizó utilizando el $\mathrm{Chi}^{2}$, encontrando que no existe diferencia en la frecuencia de presentación de los patrones de cierre entre los voluntarios sanos y los pacientes con paladar hendido. En pacientes sanos encontramos con mayor frecuencia el patrón circular (56\% de la muestra), seguido por el coronal $(29 \%)$ y el circular con rodete de Passavant (15\%). En los pacientes con paladar hendido, igualmente prevaleció el patrón circular (39.02\%), seguido del patrón coronal (24.39\%) y el patrón circular con rodete de Passavant $(19.51 \%)$. En ninguno de los 2 grupos encontramos el patrón sagital.

En conclusión, corroboramos la presentación de 3 de los 4 patrones de cierre velofaríngeo descritos en la literatura (circular, coronal, circular con rodete de Passavant), sin que existiera una diferencia estadística en la frecuencia de presentación de los patrones de cierre velofaríngeo entre los voluntarios sanos y los pacientes con hendidura palatina. No corroboramos la presentación del patrón sagital.

\section{Palabras clave Paladar Hendido, Patrón de Cierre \\ Cierre Velofaríngeo, Videonasofaringoscopia.}

Código numérico 2312-23123-14-1422
The purpose of this study is to define the patterns of velopharyngeal closure described in the literature, and its frequency of appearance in a non-probabilistic sample with healthy volunteers and to compare these findings with the ones obtained in patients with surgically repaired cleft lip and palate.

We designed a descriptive study of cross section. The evaluations were made in a fourth level hospital and in the Rehabilitation Centre for Children with Cleft lip and Palate (FISULAB), Santa fe de Bogotá (Colombia).

One hundred healthy volunteers (university students) and 82 patients with surgically repaired cleft palate participated in the study.

Videonasopharyngoscopy was performed to determine patterns of velopharyngeal closure present and their frequency of appearance.

The statistical analysis indicate that there is no difference in the frequency of presentation of the patterns of velopharyngeal closure between healthy volunteers and the patients with cleft palate. In the healthy group, the most frequently found pattern was the circular $(56 \%$ of the sample), followed by the coronal (29\%) and circular with Passavant's ridge (15\%). In the group of patients with cleft lip and palate, the circular pattern also prevails $(39,02 \%)$, followed by the coronal pattern $(24,39 \%)$, and the circular pattern with Passavant's ridge $(19,51 \%)$. In neither group was found the sagittal pattern.

As a conclusion, there was not any statistical difference in the frequency of presentation of the patterns of velopharyngeal closure between the healthy volunteers and the patients with cleft palate.

Key words Cleft palate, Pattern of Velopharyngeal

Velopharyngeal Closure, Videonasopharyngoscopy

Numeral Code ～2312-23123-14-1422

\footnotetext{
* Cirujano Plástico FISULAB. Jefe del Servicio de Cirugía Plástica del Hospital Infantil Universitario de San José. Cirujano Plástico Fundación Santa Fe de Bogotá.

** Cirujano Plástico Hospital Infantil de San José. Cirujano Plástico Fundación Santa Fe de Bogotá.

*** Directora y Fonoaudióloga FISULAB

**** Fonoaudióloga FISULAB
}

FISULAB, Centro de Rehabilitación para Niños con Labio y Paladar Fisurado y Hospital Universitario San José. Santa Fé de Bogotá. Colombia. 


\section{Introducción}

El propósito de este trabajo es definir la frecuencia de aparición de los patrones de cierre velofaríngeo descritos en la literatura en una muestra intencional compuesta por voluntarios sanos, mediante la realización de videonasofaringoscopia y posterior comparación de los resultados con los obtenidos en pacientes con paladar fisurado reparado quirúrgicamente (1-4).

El cierre del esfínter velofaríngeo (válvula o esfínter) es fundamental para la producción del habla. Se trata de un mecanismo tridimensional que involucra al velo del paladar y a las paredes faringeas laterales y posterior. $\mathrm{Su}$ adecuado funcionamiento depende de los músculos del paladar y de la faringe, que requieren de una integridad estructural y neurológica. La función velofaríngea está relacionada con un cierre velofaríngeo total que impide durante la deglución o fonación, el paso de comida o aire a la nasofaringe (5-7).

Los músculos que principalmente intervienen en el cierre velofaríngeo son el elevador del velo del paladar (levator veli palatini), palatofaríngeo y constrictor superior de la faringe (Fig. 1) (1,5,6,8).

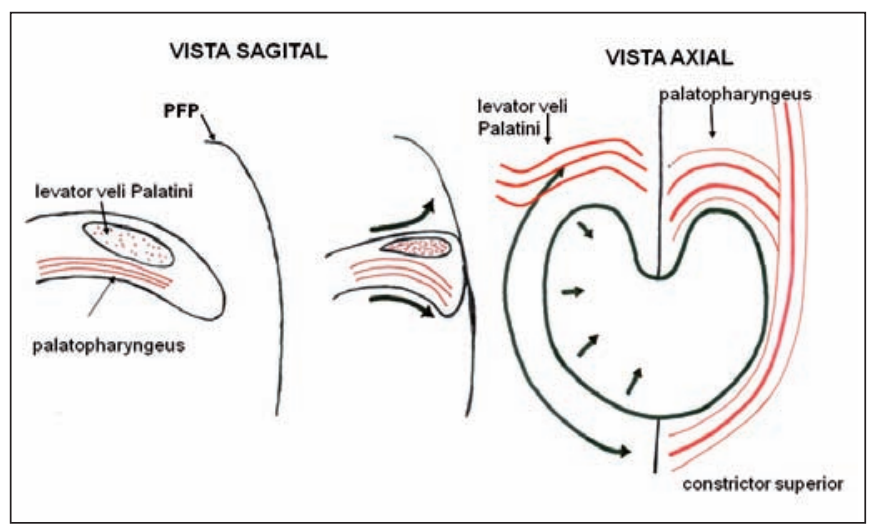

Figura 1. Vista sagital y axial del esfínter velofaríngeo donde se observa la participación de los músculos palatofaringeo, elevador y constrictor superior.

Se afirma que el músculo constrictor superior de la faringe es el responsable del componente faríngeo del cierre. Estudios electromiográficos han demostrado una actividad inconsistente del constrictor superior dependiendo del patrón de cierre de cada paciente, ya sea coronal (con mínima actividad o sin actividad), circular (actividad moderada a elevada) o sagital (actividad elevada) $(4-6,8)$.

El conocimiento de las dimensiones espaciales del esfínter velofaríngeo es la base para el entendimiento de la fisiología velofaringea, lo que es indispensable para optimizar los procedimientos quirúrgicos y la rehabilitación funcional de los pacientes con insuficiencia velofaríngea (IVF) $(3,7)$.

Son varios los estudios disponibles para valorar el cierre velofaríngeo. Se han usado métodos imagenológicos como la Tomografía Axial Computerizada (TAC) y la Resonancia Nuclear Magnética (RNM), con el inconve- niente de que las imágenes obtenidas son estáticas y no permiten la valoración dinámica y funcional del esfínter velofaríngeo (9).

La videonasofaringoscopía junto con la videofluoroscopia, están aceptadas como técnicas óptimas para la observación y valoración directa de los movimientos velofaríngeos (2-4); se puede utilizar uno o ambos métodos en combinación.

La videofluoroscopia es una técnica radiológica diseñada para valorar el cierre velofaríngeo, en la que se observan los movimientos de las estructuras involucradas en el cierre velofaríngeo en proyección lateral y ánteroposterior, durante la deglución y la fonación, utilizando un medio de contraste local. Las imágenes se graban para proveer un registro continuo que permite su análisis posterior (2). También es posible tomar otras proyecciones, pero por la superposición de estructuras óseas son más complicadas de interpretar. El hecho de someter al paciente y al examinador a radiación, así como la dificultad de contar con un equipo de radiología apropiado en los centros de labio y paladar hendido, ha hecho que la endoscopia cobre mayor popularidad entre los grupos de especialistas que atienden estos pacientes.

La videonasofaringoscopia consiste en introducir un nasofaringoscopio flexible a través de una fosa nasal, pasando a través del meato medio, lo que permite la visualización directa del esfínter velofaringeo de sus estructuras y de los movimientos realizados durante el habla y la deglución, en forma dinámica, desde una proyección superior (2,3) (Fig. 2).

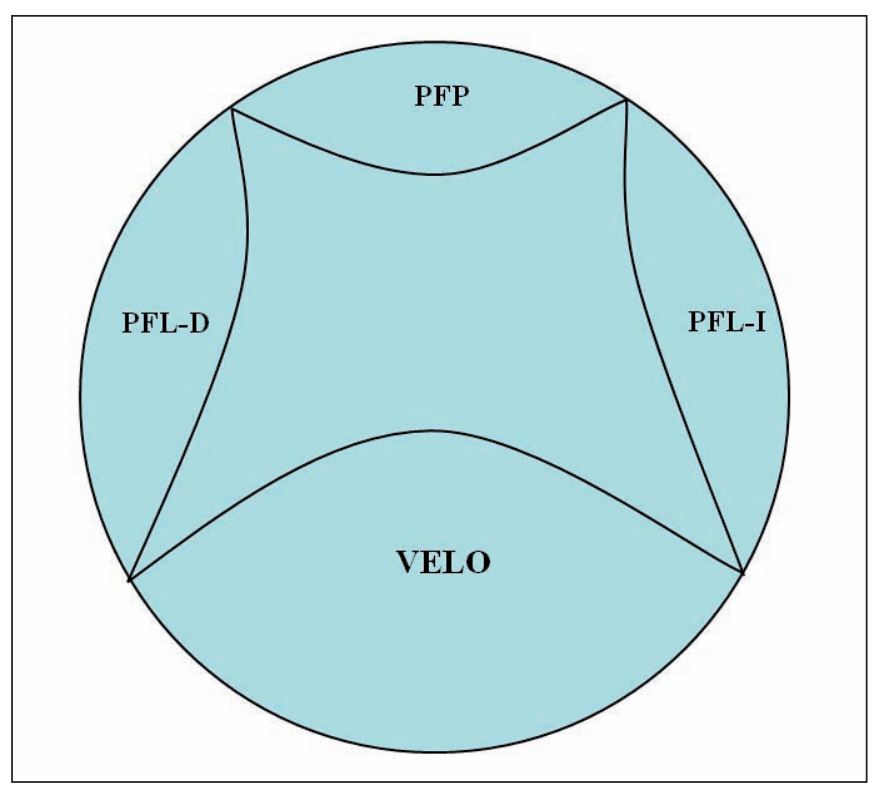

Figura 2. Esquema de la visón en pantalla durante la videonasofaringoendoscopia; observamos en la parte superior la pared faríngea posterior, a los lados las paredes faríngeas laterales y en la parte inferior el velo del paladar.

Skolnick y Croft $(10,11)$, describieron inicialmente diferentes patrones de cierre velofaríngeo. De acuerdo al movimiento de las estructuras durante la fonación es posible considerar la existencia de 4 patrones: Coronal (Fig. 3), Sagital (Fig. 4), Circular (Fig. 5) y Circular con Rodete 


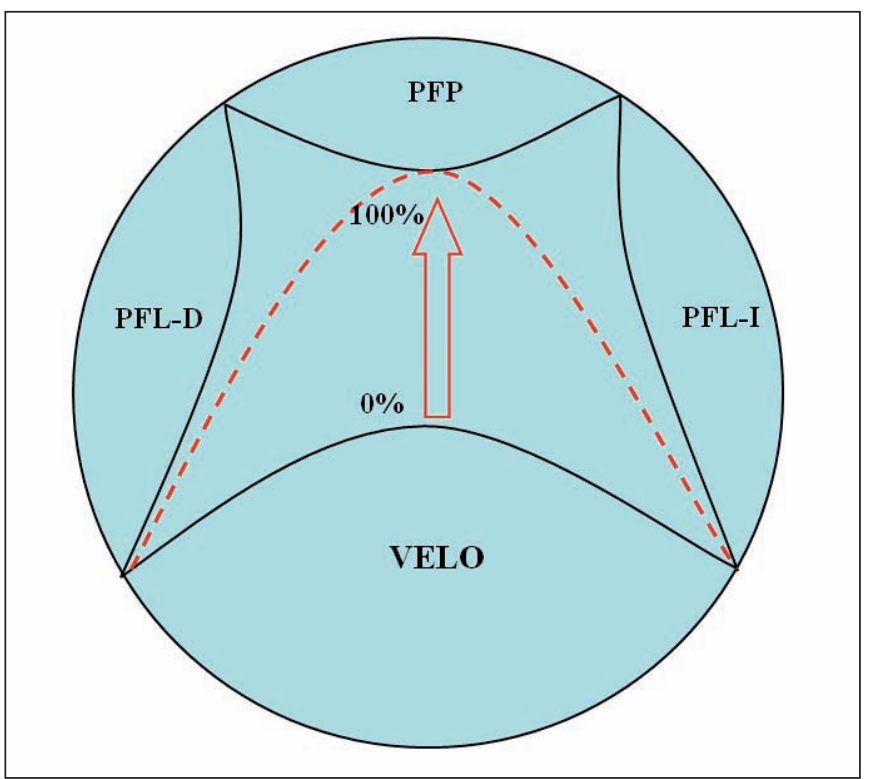

Figura 3. Patrón de cierre velofaringeo coronal.

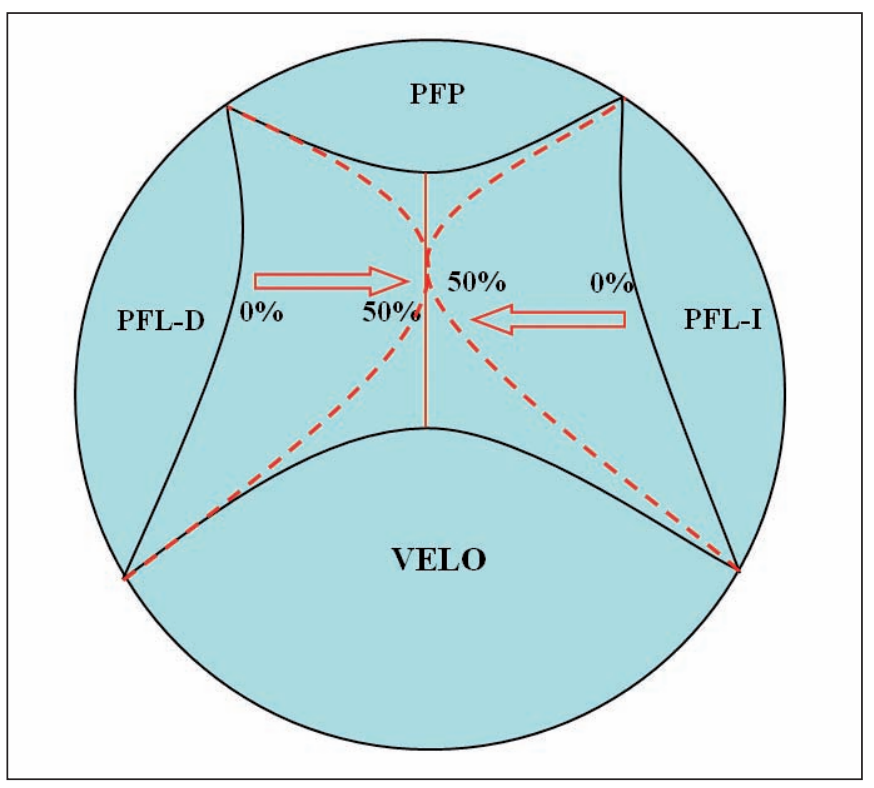

Figura 4. Patrón de cierre velofaringeo sagital.

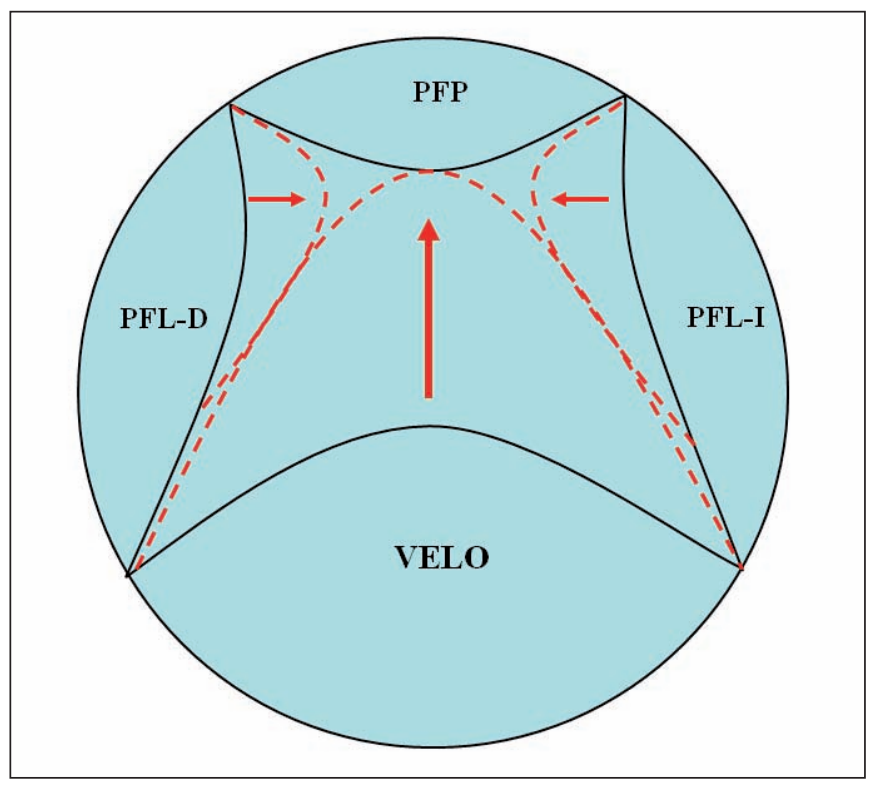

Figura 5. Patrón de cierre velofaringeo circular.

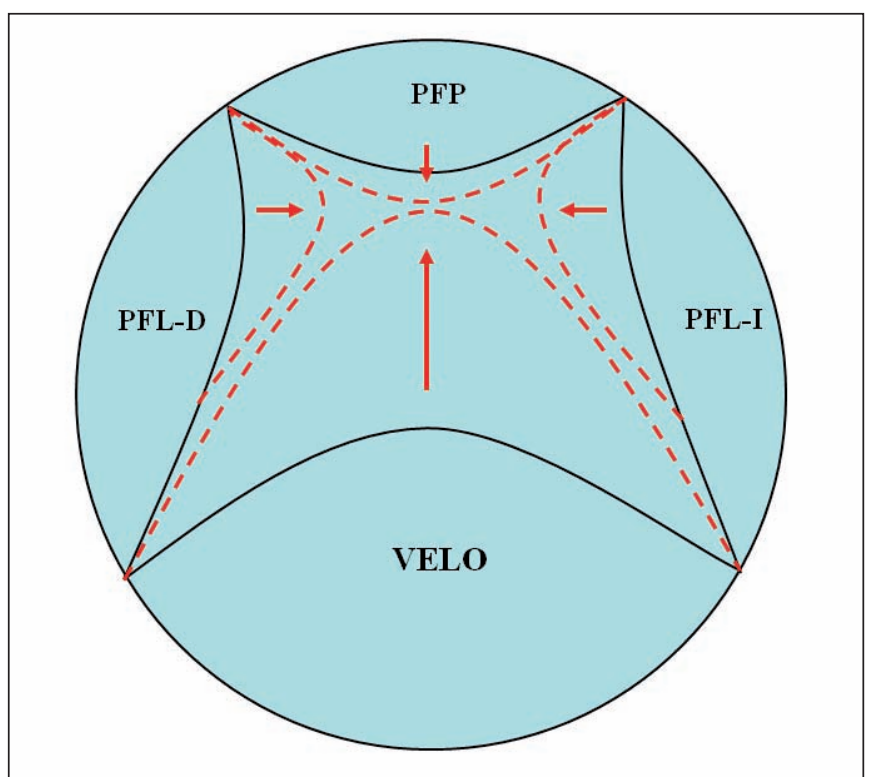

Figura 6. Patrón de cierre velofaringeo circular con rodete de Passavant.

o Anillo de Passavant (Fig. 6), según las estructuras que se muevan durante la fonación. (7).

En el patrón coronal, el cierre del esfínter se obtiene por el movimiento exclusivo del velo del paladar, que se desplaza hacia la pared faríngea posterior sin involucrar movimientos de otras estructuras y ese desplazamiento se mide en porcentaje de movimiento (Fig. 3). El patrón sagital corresponde al cierre velofaríngeo a partir del movimiento de las paredes faríngeas laterales y se mide en porcentaje de movimiento de cada lado hacia la línea media, un 50\% por lado (Fig. 4). El patrón circular es el que produce el cierre Velofaríngeo por el movimiento del velo y de las paredes faríngeas laterales y se mide según el porcentaje de movimiento de cada estructura (Fig. 5). En el Patrón Circular con Rodete se obtiene el cierre velofaríngeo por el movimiento de las cuatro paredes, es decir, es un patrón circular al que se suma el movimiento de la pared faríngea posterior a nivel del Rodete o Anillo de Passavant, el cual se mide también en porcentajes de movimiento. El movimiento de la pared faríngea posterior generalmente nunca es mayor del 10\% (Fig. 6).

Pocos son los estudios que describen el cierre velofaríngeo en poblaciones sanas (11-14) ya que la mayoría se realizan sobre pacientes con diagnostico de insuficiencia velofaríngea (IVF), generalmente asociada a patologías como labio y paladar hendido. Tampoco se conoce la frecuencia de presentación de los patrones de cierre entre la población con fisura palatina.

\section{Material y método}

Realizamos un estudio descriptivo de corte transversal, con una muestra no probabilística de 100 voluntarios sanos, 62 mujeres y 38 hombres, estudiantes de Medicina y Odontología, entre los 16 y los 40 años de edad con un promedio de 24 años. A todos se les realizó nasofarin- 
goscopia para determinar su patrón de cierre velofaríngeo, previa autorización escrita. Excluimos a aquellos voluntarios que presentaban antecedentes de cirugía orofaríngea y a los que habían presentado problemas del habla en la infancia.

A continuación realizamos el estudio en una muestra de 82 pacientes con paladar hendido corregido quirúrgicamente, 44 hombres y 38 mujeres, entre los 2 y los 25 años de edad (media de 9,46 años), que asistían al Centro de Rehabilitación para Niños con Labio y Paladar Fisurado (FISULAB) y al Hospital San José, en Santa Fe de Bogotá (Colombia).

De los niños con paladar hendido, fueron incluidos en el estudio aquellos que consultaron en los 2 centros mencionados, con secuelas de paladar hendido con o sin compromiso del labio y que hubieran tenido por lo menos una corrección quirúrgica del paladar, no sindromáticos ni con patologías asociadas y que tuvieran la edad mínima para colaborar con el examen. Se excluyeron aquellos en los que no se logró una visualización adecuada del esfínter velofaríngeo.

Los pacientes con paladar hendido se dividieron en 3 grupos: el Grupo 1 correspondía a los pacientes tratados integralmente, desde recién nacidos, en FISULAB; el Grupo 2 incluía a los que acudieron para estudio de IVF en FISULAB y en el Hospital de San José enviados desde otros centros; el Grupo 3 correspondía a pacientes tratados con anterioridad en otros centros, pero evaluados en FISULAB. Se excluyeron del grupo de estudio aquellos pacientes de los que no se contaba con los datos mínimos requeridos.

Practicamos videonasofaringoscopia a cada uno de los participantes utilizando producción de sonidos verbales orales (sílabas, palabras y frases) previamente establecidos, para determinar el patrón de cierre velofaríngeo y el porcentaje de movilidad de las estructuras. Cada uno de estos exámenes fue grabado en un videocasette y registrado por escrito en un instrumento diseñado para tal fin. Posteriormente, se recopiló toda la información en una base de datos para determinar la frecuencia de aparición de cada uno de los patrones. Utilizamos un nasoendoscopio Pentax FNL 10RP3® de 2,4 mm., cámara Endovision XL marca Karl Storz ${ }^{\circledR}$ 20280101 y una fuente de Luz Karl Storz® 20112301.

La muestra de habla recogida consistió en secuencias de monosílabos (consonante - vocal), bisílabos y frases cortas con consonantes orales que producen cierre completo del esfínter (explosivas y fricativas) (Tabla I).

La planilla utilizada como instrumento de recolección de la información contenía datos de identificación de los pacientes, registro del patrón de cierre y una figura circular basados en la estandarización para registros de nasofaringoscopia, dividida en cuadrantes para marcar el porcentaje de movimiento de cada una de las paredes hasta completar el $100 \%$ en la dimensión ántero-posterior y / o lateral (Fig. 7).

Adicionalmente se escribieron como observaciones
Tabla I. Muestra de habla

\begin{tabular}{|c|c|c|c|c|}
\hline \multirow[t]{4}{*}{ Monosílabos } & $\mathrm{PA}$ & PE & PI & $\mathrm{PO}$ \\
\hline & KA & $\mathrm{KE}$ & KI & $\mathrm{KO}$ \\
\hline & TA & TE & TI & TO \\
\hline & SA & SE & SI & SO \\
\hline \multirow[t]{2}{*}{ Bisílabos } & PAPO & GATO & SAKO & GATA \\
\hline & TITO & PATO & TACO & KIKO \\
\hline \multirow[t]{5}{*}{ Frases cortas } & PEPE PICA PAPA & & & \\
\hline & LA COLA DEL PATO & & & \\
\hline & TITO TOCA TETERO & & & \\
\hline & SUSI SALE SOLA & & & \\
\hline & KUKI KOGE KOKO & & & \\
\hline
\end{tabular}

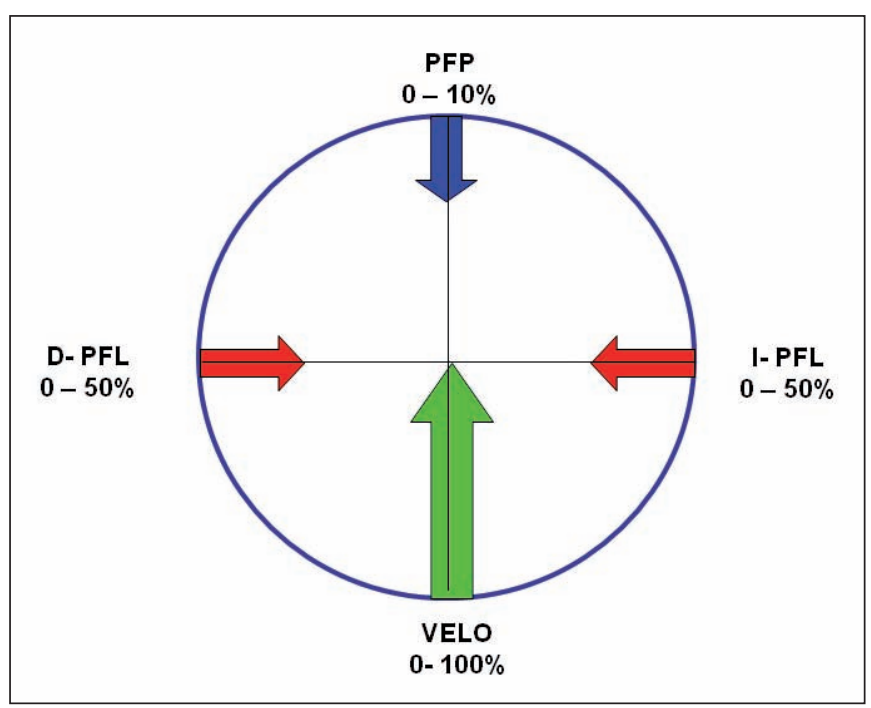

Figura 7. . Porcentaje de movimiento de las paredes faríngeas y del velo del paladar.

cualquier situación temporal o permanente que afectara al estado o funcionalidad de la válvula velofaríngea, como cirugías o enfermedades en la oro o nasofaringe, estados gripales o alteraciones del habla, con el fin de analizar posteriormente si esto afectaba o no a la imagen obtenida. Para los pacientes con secuelas de paladar hendido, se revisaron algunos datos de la historia clínica, como el tipo de hendidura y el número y tipo de cirugías realizado en el paladar o la faringe. Se realizaron también pruebas de concordancia intra e interexaminador con niveles aceptables en el grupo inicial de pacientes sanos (kappa interobservador $70 \%$; intraobservador $80 \%$; $95 \%$ confianza).

El plan de análisis se desarrolló en 2 fases. La primera consistió en realizar análisis univariado de los principales indicadores clínicos y sociodemográficos registrados en tablas de frecuencia cuando las variables eran de naturaleza categórica y promedios y desviación estándar para variables de tipo continuo. Se tuvieron en cuenta la edad y el género, tanto de la población sana como de la población con hendidura. Para esta última, se investigó además el tipo de hendidura y las cirugías realizadas en el paladar, tanto en número como en técnica quirúrgica empleada.

Posteriormente, hicimos un análisis bivariado teniendo en cuenta el género y la edad para el grupo al cual pertenecía el 
paciente. También se analizó el patrón de cierre velofaringeo de acuerdo al diagnóstico, al grupo al cual pertenecen y al tipo de cirugía realizada. Finalmente se investigó si había relación entre IVF, el grupo y el tipo de patrón.

Exploramos las diferencias en proporciones de variables categóricas según indicadores sociodemográficos mediante pruebas de $\mathrm{Chi}^{2}$ y test exacto de Fisher (valor $\mathrm{p}<0.05$ ). Todos los análisis estadísticos se llevaron a cabo con el programa SAS (SAS Institute, Cary NC USA)

\section{Resultados}

De las 182 videofaringoscopias realizadas, 100 correspondían a los voluntarios sanos y 82 a pacientes con secuelas de paladar hendido. De estos últimos, 22 pacientes pertenecían al grupo 1 (pacientes atendidos en FI-
SULAB desde su nacimiento): 14 hombres y 8 mujeres; 9 con hendidura bilateral, 12 unilateral y 1 con paladar hendido, con edad promedio de 6,59 años.

$\mathrm{Al}$ grupo 2 pertenecieron 12 pacientes, remitidos para estudio nasofaringoscópico por IVF: 5 hombres y 7 mujeres; 3 con hendidura bilateral, 5 con hendidura unilateral y 4 con paladar hendido, con edad promedio de 8,75 años.

Los del Grupo 3 (pacientes atendidos inicialmente en otras instituciones) fueron 48 pacientes: 25 hombres y 23 mujeres; 20 con hendidura bilateral, 21 con hendidura unilateral y 7 con paladar hendido; con una edad promedio de 13,04 años. En total, 32 pacientes con hendidura bilateral, 38 unilateral, siendo más frecuente la fisura izquierda (26 izquierda y 12 derecha) y 12 con paladar hendido solamente, desde completo hasta submucoso (Tabla II).

Tabla II. Análisis de diagnóstico por grupo de procedencia del paciente, género y edad

\begin{tabular}{|c|c|c|c|c|c|c|c|}
\hline Grupo & Bilateral & Unilateral & PH & Total & $\begin{array}{l}\text { \% por } \\
\text { Grupo }\end{array}$ & $\begin{array}{l}\text { Promedio } \\
\text { de edad }\end{array}$ & DS \\
\hline Grupo 1 & $9: 5 m-4 f$ & $12: 9 m-3 f$ & 1: $\quad$ if & $22: 14 m-8 f$ & 26,82 & 6,59 & 2,06 \\
\hline Grupo 2 & $3.1 \mathrm{~m}-2 \mathrm{f}$ & $5: 3 m-2 f$ & $4: 1 m-3 f$ & $12: 5 \mathrm{~m}-7 \mathrm{f}$ & 14,63 & 8,75 & 5,81 \\
\hline Grupo 3 & 20: $11 \mathrm{~m}-9 \mathrm{f}$ & $21: 12 \mathrm{~m}-9 \mathrm{f}$ & $7: 2 m-5 f$ & $48: 25 m-23 f$ & 58,53 & 13,04 & 4,45 \\
\hline TOTAL & $32: 17 m-15 f$ & $38: 24 m-14 f$ & $12: 3 m-9 f$ & $82: 44 m-38 f$ & & & \\
\hline$\%$ por tipo de hendidura & 39,02 & 46,34 & 14,63 & 100 & & & \\
\hline
\end{tabular}

m: masculino

f: femenino

DS: Diferencia significativa

En el grupo de sanos, encontramos $56(56 \%)$ con patrón circular (19 hombres y 37 mujeres), 29 (29\%) coronal (11 hombres y 18 mujeres) y 15 (15\%) circular con rodete ( 8 hombres y 7 mujeres). De los pacientes con hendidura, $32(39,02 \%)$ presentan patrón circular $(24$ hombres, 8 mujeres), 20 patrón coronal $(24,39 \%$ ) (8 hombres y 12 mujeres) y 16 patrón circular con rodete
$(19,51 \%)$ (6 hombres y 10 mujeres). En 14 pacientes $(17,07 \%)$ (6 hombres y 8 mujeres) su última cirugía de paladar fue una faringoplastia tipo Orticochea (Orticochea, 1968) (15), por lo tanto, en la imagen endoscópica, se observó un movimiento característico de la técnica que no se puede clasificar como ninguno de los patrones anteriormente descritos (Tabla III).

Tabla III. Comparación de patrones de cierre velofaríngeo en pacientes con paladar hendido según el diagnóstico y en voluntarios sanos. Comparación de patrones por género, en ambas poblaciones unidas.

\begin{tabular}{|l|c|c|c|c|c|c|c|c|}
\hline & CORONAL & $\%$ & CIRCULAR & $\%$ & $\begin{array}{c}\text { CIRCULAR + } \\
\text { RODETE }\end{array}$ & ESFINTER & \% \\
\hline $\begin{array}{l}\text { PACIENTES CON } \\
\text { HENDIDURA }\end{array}$ & $20: 8 \mathrm{M}-12 \mathrm{~F}$ & 24,39 & $32: 24 \mathrm{M}-8 \mathrm{~F}$ & 39,02 & $16: 6 \mathrm{M}-10 \mathrm{~F}$ & 19,51 & $14: 6 \mathrm{M}-8 \mathrm{~F}$ & 17,07 \\
\hline LPH BILATERAL & $9: 3 \mathrm{M}-6 \mathrm{~F}$ & & $10: 7 \mathrm{M}-3 \mathrm{~F}$ & & $6: 4 \mathrm{M}-2 \mathrm{~F}$ & & $7: 3 \mathrm{M}-4 \mathrm{~F}$ & $6: 3 \mathrm{M}-3 \mathrm{~F}$ \\
\hline LPH UNILATERAL & $10: 5 \mathrm{M}-5 \mathrm{~F}$ & & $18: 15 \mathrm{M}-3 \mathrm{~F}$ & & $4: 1 \mathrm{M}-3 \mathrm{~F}$ & & $1: 0 \mathrm{M}-1 \mathrm{~F}$ & \\
\hline PH AISLADO & $1: 0 \mathrm{M}-1 \mathrm{~F}$ & & $4: 2 \mathrm{M}-2 \mathrm{~F}$ & & $6: 1 \mathrm{M}-5 \mathrm{~F}$ & & 0 \\
\hline $\begin{array}{l}\text { VOLUNTARIOS } \\
\text { SANOS }\end{array}$ & $29: 11 \mathrm{M}-18 \mathrm{~F}$ & 29 & $56: 19 \mathrm{M}-37 \mathrm{~F}$ & 56 & $15: 8 \mathrm{M}-7 \mathrm{~F}$ & 15 & 0 \\
\hline $\begin{array}{l}\text { TOTAL DE LA } \\
\text { POBLACIÓN }\end{array}$ & $49: 19 \mathrm{M}-30 \mathrm{~F}$ & 26,92 & $88: 43 \mathrm{M}-45 \mathrm{~F}$ & 48,35 & $31: 14 \mathrm{M}-17 \mathrm{~F}$ & 17,03 & $14: 6 \mathrm{M}-8 \mathrm{~F}$ & 7,69 \\
\hline
\end{tabular}


Realizamos un análisis de $\mathrm{Chi}^{2}$ comparando la alteración anatómica y el patrón de cierre. Se incluyeron los pacientes que presentaban fisura bilateral, fisura unilateral y los pacientes con paladar hendido aislado y utilizamos el programa Stata 9,1. Inicialmente analizamos los 3 diagnósticos con los 3 patrones presentados, encontrando un resultado de 9,2107 para una p - 0,056. Para verificar dónde se encontraba la diferencia, analizamos los grupos entre sí. Analizando los pacientes con hendidura bilateral y los pacientes con hendidura unilateral, obtuvimos un valor 1,9075 para una $\mathrm{p}$ - 0,385. Comparando los pacientes con hendidura bilateral con los pacientes que solo presentaban paladar hendido, obtuvimos un valor de 4,1554 para una $\mathrm{p}$ - 0,125. Comparando los pacientes con fisura unilateral con los pacientes con hendidura palatina, obtuvimos un valor de 8,4268 con una p - 0,015. Esto demostró que la diferencia estadísticamente significativa entre los grupos se daba al comparar los pacientes con fisura unilateral con los de paladar hendido aislado.

Todos los patrones coronales en voluntarios sanos tuvieron un movimiento del 100\% del velo, a excepción de uno que presentó un movimiento del 95\%, no siendo así para los pacientes con hendidura en los cuales se encontraron patrones coronales insuficientes, con un porcentaje de movimiento entre el 5 y el $100 \%$.

Dentro de los patrones circulares, el porcentaje de movilidad de las paredes faríngeas laterales osciló entre $10 \%$ y $50 \%$, tanto para los voluntarios sanos como para los pacientes con hendidura.

En los patrones circulares con rodete, el porcentaje del movimiento del rodete varió entre un 5\% y un $10 \%$, tanto para los voluntarios sanos como para la mayoría de los pacientes con hendidura. En un paciente con hendidura se observó un movimiento del $15 \%$.

En uno de los voluntarios evaluados y en un paciente con hendidura con patrón circular, encontramos asimetría en el movimiento de sus paredes faríngeas laterales (35\% y $15 \%$ ),

No encontramos ningún patrón sagital, ni entre los voluntarios sanos, ni entre los pacientes con hendidura (Tabla III).

Realizamos un análisis de $\mathrm{Chi}^{2}$ utilizando el programa Epi Info versión 332 para verificar si se encontraba diferencia estadística entre los patrones de cierre en los voluntarios sanos, comparados con los pacientes con paladar hendido. Encontramos un resultado de 2,22 con un valor $\mathrm{p}$ - 0,33022990, comprobando que no había diferencia entre estos grupos.

Según el grupo al que pertenecen los pacientes, encontramos un predominio de pacientes con patrón coronal en el Grupo 1 (10 pacientes, 45,4\%), seguidos por los pacientes con patrón circular (7 pacientes, $31,81 \%$ ) y circular con rodete (4 pacientes, 18,18\%). Solo se encontró 1 paciente $(4,54 \%)$ con esfínter. En el Grupo 2, predominaron los patrones circulares con rodete (5 pacientes, $41,66 \%$ ), seguidos del patrón coronal (3 pacientes, $25 \%$ ) y en número igual por el patrón circular y esfínter ( 2 pacientes, 16,66\%). En el Grupo 3 predominó el patrón circular (23 pacientes, $47,91 \%$ ), seguido por pacientes con esfínter (11 pacientes, $22,91 \%$ ) y en igual número por pacientes con patrones coronal y circular con rodete (7 pacientes, $14,58 \%$ ) (Tabla IV).

Tabla IV. Patrones de cierre velofaríngeo en pacientes con paladar hendido de acuerdo al grupo de procedencia y en voluntarios sanos.

\begin{tabular}{|l|c|c|c|c|c|c|c|c|c|}
\hline & CORONAL & $\boldsymbol{\%}$ & CIRCULAR & $\boldsymbol{\%}$ & $\begin{array}{c}\text { CIRCULAR + } \\
\text { RODETE }\end{array}$ & $\boldsymbol{\%}$ & ESFINTER & \% & TOTAL \\
\hline Grupo 1 & 10 & 45,45 & 7 & 31,81 & 4 & 18,18 & 1 & 4,54 & 22 \\
\hline Grupo 2 & 3 & 25 & 2 & 16,66 & 5 & 41,66 & 2 & 16,66 & 12 \\
\hline Grupo 3 & 7 & 14,58 & 23 & 47,91 & 7 & 14,58 & 11 & 22,91 & 48 \\
\hline TOTAL & 20 & 24,39 & 32 & 39,02 & 16 & 19,51 & 14 & 17,07 & 82 \\
\hline
\end{tabular}

Uno de los voluntarios sanos evaluados que presentaba un patrón circular, presentó una IVF leve (5\%) que se evidenció clínicamente por una ligera nasalidad que no afectaba a su comunicación. Por el contrario, 35 $(42,68 \%)$ de los pacientes con hendidura, presentaron IVF, siendo éste su principal problema.

Teníamos la intención inicial de determinar si el patrón presente se relacionaba de alguna manera con la técnica quirúrgica realizada; sin embargo, solo 33 (17 del Grupo 1: 77,27\%, 2 del Grupo 2: 16,66\% y 14 del Grupo 3: $29,16 \%$ ) de los 82 pacientes con hendidura, tenían en el momento de la evaluación una sola intervención quirúrgica en el paladar, reportada como cirugía 1. Los 49 restantes, tenían cirugías posteriores, registradas como cirugía 2 y cirugía 3 . De los pacientes con una sola cirugía del Grupo 1, 4 (23,52\%) presentan IVF; del Grupo 2, $2(100 \%)$ presentaba IVF y del grupo $3,8(57,14 \%)$.

Además, solo en el Grupo 1 teníamos control sobre el cirujano y las técnicas quirúrgicas utilizadas, ya que por el origen de los pacientes de los Grupos 2 y 3 , era difícil determinar quién había sido el cirujano y qué técnicas quirúrgicas se utilizaron puntualmente para cada intervención. Solo se podía estudiar el Grupo 1 en cuanto a la comparación de las técnicas quirúrgicas y del patrón de cierre.

Comparamos la presencia o no de insuficiencia velofaríngea de acuerdo al patrón de cierre, sin encontrar diferencia alguna (Tabla V). 
Tabla V. Presencia de insuficiencia velofaríngea (IVF) de acuerdo al patrón de cierre presentado.

\begin{tabular}{|l|c|c|c|c|c|c|c|c|}
\hline $\begin{array}{c}\text { INSUFICIENCIA } \\
\text { VELOFARINGEA (IVF) }\end{array}$ & CORONAL & $\boldsymbol{\%}$ & CIRCULAR & $\boldsymbol{\%}$ & $\begin{array}{c}\text { CIRCULAR + } \\
\text { RODETE }\end{array}$ & $\boldsymbol{\%}$ & ESFINTER & $\boldsymbol{\%}$ \\
\hline PRESENTE & 9 & 45 & 16 & 50 & 8 & 50 & 3 & 21,42 \\
\hline AUSENTE & 11 & 55 & 16 & 50 & 8 & 50 & 11 & 78,57 \\
\hline & 20 & & 32 & & 16 & 5 & 14 & \\
\hline
\end{tabular}

\section{Discusión}

Determinamos los patrones de cierre velofaríngeo en una muestra intencional con voluntarios pertenecientes a población colombiana sana, lo que arrojó información que hasta la fecha no había sido estudiada. En la literatura mundial son contadas las referencias de estudios hechos sobre patrones en voluntarios sanos (14).

Después de realizar el análisis estadístico, no encontramos ninguna diferencia entre los pacientes con paladar hendido y la población sana, relacionada con los patrones de cierre velofaríngeo presentados o con su porcentaje de aparición.

Confirmamos la existencia de 3 de los 4 patrones descritos por Skolnick y Croft $(10,11)$, lo cual se correlaciona igualmente con los hallazgos de los estudios anatómicos descritos (6). No se encontró ningún patrón Sagital puro, aunque en 3 de los casos registrados como patrón circular, dado el movimiento tan fuerte de las paredes laterales (del 40 a $50 \%$ ) y con un movimiento relativamente menor del velo (50 a 60\%), se podría interpretar erróneamente como un componente sagital o un nuevo patrón. Esto puede considerarse importante, dado que la mayoría de los estudios le atribuyen un componente prioritario al musculo levator veli palatini $(6$, 12), el cual estaría prácticamente inactivo en los patrones sagitales puros. Si en estos casos se valorara exclusivamente la fluoroscopia en la proyección anteroposterior se interpretaría como un patrón sagital.

Como dato curioso, referimos que los investigadores en su experiencia personal de más de 14 años con la utilización de la videonasofaringoscopia, solo han visto 2 casos de patrón de cierre sagital, uno en un paciente con labio y paladar hendido y otro en un paciente con insuficiencia velofaríngea por faringe profunda y grande (caja amplia).

En el patrón circular con rodete, observamos que los pacientes con hendidura palatina tienden a desarrollar un mayor movimiento del rodete, tanto en desplazamiento como en intensidad, comparado con los voluntarios sanos. De hecho, en estos últimos prima un movimiento del 5\% (60\%) mientras que en los pacientes con hendidura no solo prima un movimiento del $10 \%$ (62,5\%), sino que en uno de los casos se encontró movimiento del $15 \%$.

Comparando el porcentaje de presentación de los patrones en los diferentes grupos, observamos una tenden- cia a la presentación del patrón coronal en el Grupo 1; sin embargo, por la distribución de los mismos, no son grupos comparables entre sí (Tabla IV).

Comparando la presencia de IVF según el patrón de cierre, no observamos ninguna tendencia hacia algún patrón en particular (Tabla V). Sin embargo, es fundamental resaltar la importancia de determinar el tipo de patrón presente en los casos de IVF, con el fin de planear el tratamiento quirúrgico corrector en base a dichos hallazgos $(3,4)$.

En el presente estudio queríamos evidenciar el funcionamiento velofaríngeo desde una perspectiva puramente anatómica y funcional, determinando los distintos patrones de cierre, a diferencia de la mayoría de publicaciones que apuntan al estudio de su funcionamiento partiendo del análisis aerodinámico y electroacústico, utilizando distintos instrumentos adicionales $(7,13)$.

Es importante continuar con estudios de este tipo en diferentes regiones del país para determinar si el origen racial y/o las características lingüísticas regionales influyen en los patrones de cierre velofaríngeo. En el presente trabajo, el $96 \%$ de los participantes voluntarios sanos y pacientes con hendidura provienen de la ciudad de Bogotá (Colombia).

\section{Conclusiones}

De los 4 patrones reportados en la literatura (coronal, circular, circular con rodete y sagital), solo observamos en nuestros estudios patrón coronal, circular y circular con rodete. No observamos ningún caso de patrón sagital.

El patrón de cierre tiene una misma distribución en pacientes sanos comparados con pacientes de paladar hendido.

Por la distribución de los grupos de estudio de los pacientes con fisura palatina, no se puede determinar si el tipo de fisura, la cirugía practicada y el género, influyen sobre el tipo de patrón de cierre.

Este estudio será utilizado como base para otros 2 estudios posteriores: uno en diferentes regiones del país en pacientes sanos con el fin de corroborar si hay diferencias según el patrón linguiístico, y otro en el que se estudiarán grupos étnicos cerrados para valorar las diferencias raciales.

Pensamos que todo esto será de utilidad en el enfoque terapéutico de los pacientes con paladar hendido que presentan insuficiencia velofaríngea. 


\section{Agradecimientos}

A los estudiantes de la Facultad de Medicina Fundación Universitaria Ciencias de la Salud, la Facultad de Odontología Universidad del Bosque y a los pacientes de los diferentes grupos que colaboraron para esta investigación.

\section{Dirección del autor}

Dr. José Rolando Prada Madrid

Calle $120 \mathrm{~N}^{\circ} 7$-79. Bogotá, Colombia.

e-mail: rpradam@gmail.com

\section{Bibliografía}

1. 1. David, JD, Bagnall, AD.: "Velopharyngeal Incompetence" McCarthy. Plastic Surgery, W.B. Saunders Company; Philadelphia 1990.Vol 4,Pp: 2903 - 2921.

2. Golding-Kushner, KJ et al.: "Standarization for the Reporting of Nasopharyngoscopy and Multiview Videofluoroscopy: A Report from an International Working Group". Cleft Palate J. 1990; 27: 337.

3. Prada R, Cárdenas I, Echeverri MP, González MC, Sánchez MC, Tavera MC.: "Detección y manejo temprano de la Insuficiencia Velofaríngea: Una Propuesta de manejo integral”. Rev Colom Cir Plast y Recons. 2003; 9: 20 .

4. Ysunza A, Pamploma MC, Molina F, Chacon E, Collado M.: "Velopharyngeal Motion after Sphincter Pharyngoplasty: A Videonasopharyngoscopic and Electromyographic Study". Plast Rec. Surg. 1999; 104: 905.

5. Gray H.: "Anatomy of the Human Body". 20 Edition, New York: Bartleby.com; 2000. Capítulo 11, Pp: 1141 1143.
6. Huang M, Lee S, Rajendran K.: "Anatomic Basis of Cleft Palate and Velopharyngeal Surgery: Implications from a Fresh Cadaveric Study". Plast Rec. Surg. 1998; 101: 613.

7. Poppelreuter S, Engelke W, Bruns T.: "Quantitative Analysis of the Velopharyngeal Sphincter Function During Speech". Cleft Palate Craniofacial J. 1999; 37: 157.

8. Sadove, M., Eppley, B.: "Velopharyngeal Incompetence". Bentz, M. L., Pediatric Plastic Surgery, Appleton \& Lange; Stanford, Connecticut Edicion 1998, Capítulo 7, Pp: $121-128$.

9. Shinagawa H, Ono T, Honda E, Masaki S, Shimada Y, Fujimoto I, Sasaki T, Iriki A, Ohyama K.: "Dynamic Analysis of Articulatory Movement Using Magnetic Resonance Imaging Movies: Methods and Implications in Cleft Lip and Palate". Cleft Palate Craniofacial J. 2005; $42: 225$.

10. Skolnick ML, McCall GN, Barnes M.: "The Sphincteric Mechanism of Velopharyngeal Closure”. Cleft palate J. 1973. 10:286.

11. Croft CB, Shprintzen RJ, Ruben SJ.: "Patterns of Velopharyngeal Valving in Normal and Cleft Palate Subjects: a Multi View Videofluoroscopic and Nasoendoscopic Study”. Laryngoscope. 1981; 91: 265.

12. Kuehn D, Moon J.: "Velopharyngeal Closure Force and Levator Veli Palatini Activation Levels in Varying Phonetic Contexts". Journal of Speech, Language, and Hearing Research. 1998; 41: 51.

13. Leeper H, Tissington L, Munhall K.: "Temporal Characteristics of Velopharyngeal Function in Children". Cleft Palate Craneofacial J. 1998; 35: 215.

14. Wu J T, Noordhoff M S.: "Nasopharyngoscopic analysis of the velopharyngeal function in normal and cleft lip and palate patients". Changgeng Yi Xue Za Zhi. 1996; 19(4):305.

15. Orticochea M.: "Construction of a dynamic muscle sphincter in cleft palate”. Plast Rec. Surg. 1968; 41:323. 